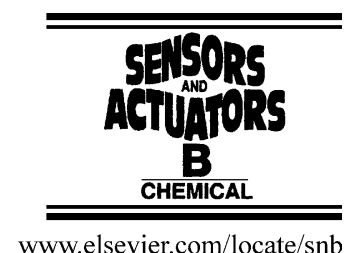

\title{
Enhancing chemiresistor-type NO gas-sensing properties using ethanol-treated lead phthalocyanine thin films
}

\author{
Kuo-Chuan $\mathrm{Ho}^{\mathrm{a}, \mathrm{b}, *}$, Chun-Ming Chen ${ }^{\mathrm{a}}$, Jung-Yu Liao ${ }^{\mathrm{a}}$ \\ ${ }^{a}$ Department of Chemical Engineering, National Taiwan University, Taipei 10617, Taiwan \\ ${ }^{\mathrm{b}}$ Institute of Polymer Science and Engineering, National Taiwan University, Taipei 10617, Taiwan
}

Received 13 July 2004; received in revised form 30 November 2004; accepted 20 December 2004

Available online 9 February 2005

Revised manuscript No. 3P111 presented at the 10th International Meeting on Chemical Sensors, July 11-14, 2004, Tsukuba, Japan

\begin{abstract}
The gas-sensing properties of ethanol-treated lead phthalocyanine ( $\mathrm{PbPc})$ thin films, to be used in a chemiresistor-type nitric oxide (NO) gas sensor, are presented. The gas-sensing properties, including current transient, sensitivity, and response time, were studied. It was found in this study that the $\mathrm{PbPc}$ thin films post-treated with an ethanol (EtOH) vapor responded faster to NO gas both in adsorption and desorption processes, comparing to those PbPc thin films without any post-treatment. The sensitivity was also enhanced with the post-treatment. A nonlinear diffusion-adsorption model, proposed in the literature for gas-sensing with a semiconductor thin film, has been used to fit our experimental data. The results obtained from the fitting revealed that the EtOH-treated $\mathrm{PbPc}$ thin films possess a larger effective diffusion coefficient and a faster response time. For $100-\mathrm{nm} \mathrm{PbPc}$ thin films, the power indexes (sensitivity) for both untreated and treated films are 0.38 and 0.65 , respectively. Moreover, the model also gives other physical parameters for both untreated and EtOH-treated $\mathrm{PbPc}$ films.
\end{abstract}

(C) 2005 Elsevier B.V. All rights reserved.

Keywords: Lead phthalocyanine; Nitric oxide sensor; Nonlinear diffusion; Solvent treatment

\section{Introduction}

The metal phthalocyanine (MPc), formed by substitution of $\mathrm{H}$ atoms in the center of phthalocyanine ring with transition metals, is a chemically and thermally stable semiconductor. Many applications have been identified, which include electrochromic device (ECD) [1,2], CD-R recording medium, solar cell [3], and gas sensing [4-24]. The p-type semiconducting lead phthalocyanine $(\mathrm{PbPc})$ was reported to be sensitive to many gases, such as $\mathrm{NO}_{2}$ [4-15], $\mathrm{NH}_{3}$ [6], $\mathrm{Cl}_{2}$ [15-17], $\mathrm{I}_{2}$ [17], $\mathrm{O}_{2}$ [18], and $\mathrm{H}_{2}$ [19]. Other p-type MPcs, such as $\mathrm{CuPc}$ and NiPc, were reported to be sensitive to both $\mathrm{NO}_{2}[6,7,10,21-23]$ and $\mathrm{NO}$ [24]. In the chemiresistor-type sensor system, the conductivity increases when a p-type semiconductor was exposed in an oxidizing

\footnotetext{
* Corresponding author. Tel.: +88622366 0739; fax: +886223623040.

E-mail address: kcho@ntu.edu.tw (K.-C. Ho).
}

gas (good electron acceptor) such as $\mathrm{NO}$ and $\mathrm{NO}_{2}$, because of the lack of electrons in a hole-carrying matrix.

Among the literature, few were mentioned about the use of MPc films for sensing NO gas, which depletes the ozone layer in the atmosphere and normally coexists with $\mathrm{NO}_{2}$ gas formed in high-temperature and -pressure furnace, with optimized preparation conditions such as morphology and post-treatment. Liu et al. [20] reported that the post-annealing temperature changes surface morphology and sensing response of a nickel phthalocyanine (NiPc) film. This implies that post-treatment can be a very effective tuning technique to obtain a stable and reproducible sensing characteristic. Furthermore, an unsteady-state response versus time relationship is also proposed by considering a reversible adsorption/desorption process between $\mathrm{NiPc}$ and $\mathrm{NO}$ gas molecule [20]. Besides understanding the transient behavior during sensing process, the relationship between the response current and the concentration of NO (calibration curve) 
is another key step to understand the PbPc-NO sensing system, which has been hardly mentioned. Therefore, the effect of post-treatment (solvent-treatment) on a PbPc thin film and the proposed mechanism based on a nonlinear diffusion-adsorption model for sensing NO gas will be discussed.

\section{Experimental}

The lead phthalocyanine $(\mathrm{PbPc}$ ) purchased (from Aldrich, $80 \%$ ) is further purified by vacuum sublimation process at $500{ }^{\circ} \mathrm{C}$ for three times. Then purified $\mathrm{PbPc}$ powder is thermally evaporated under $2.4 \times 10^{-3} \mathrm{~N} / \mathrm{m}^{2}$ vacuum onto $\mathrm{Al}_{2} \mathrm{O}_{3}$ substrates where an interdigitated gold electrode (Fig. 1) with 0.2 -mm electrode spacing is screen-printed using gold paste and heated at $930{ }^{\circ} \mathrm{C}$ for $120 \mathrm{~min}$. Film thickness $(100,200$, and $300 \mathrm{~nm})$ and evaporation rate $(0.5 \mathrm{~nm} / \mathrm{s})$ are monitored and controlled by a quartz crystal oscillator installed inside the vacuum chamber. The $\mathrm{Al}_{2} \mathrm{O}_{3}$ substrate temperature is $25^{\circ} \mathrm{C}$ during the evaporation process. The deposited $\mathrm{PbPc}$ thin film is placed in a sealed solvent vapor environment reaching equilibrium at room temperature for $24 \mathrm{~h}$. The selected solvents are ethanol, water, acetone, acetonitrile, isopropanol, and $n$-hexane. The conductance response of the film, which varies with different concentrations of NO gas tuned by mixing $500 \mathrm{ppm} \mathrm{NO}$ with ultrapure $\mathrm{N}_{2}$ gas stream from tank (SanFu Gas Co., 99.9995\%) through a flow meter, is obtained by applying a 10-V DC across both terminals of the electrodes, as shown in Fig. 1. The current responses are recorded by a potentiostat (Keithley 236). The sensing film that faces against the gas inlet at a flow rate of $200 \mathrm{ml} / \mathrm{min}$ is placed in the sensing chamber. Detailed experimental setup can be found in the previous work [24,25]. The XRD analysis is obtained by a diffractometer (MAC Science, M03XHF). The SEM pictures are taken by a scanning electron microscope (Hitachi S-800 and S-4000).

\section{Nonlinear diffusion/adsorption theory}

The general diffusion equation involving adsorption which is relatively rapid compared with diffusion process

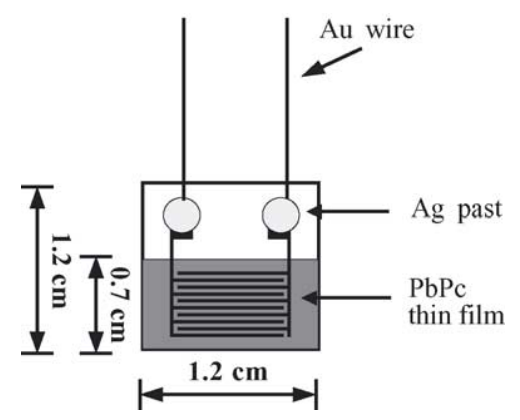

Fig. 1. The 10-fingered interdigitated sensing electrode.

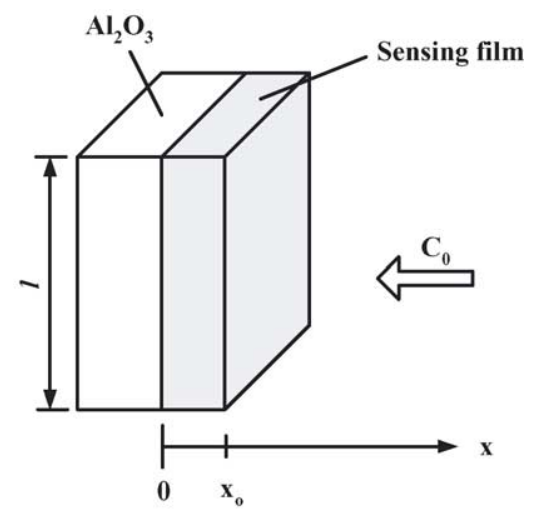

Fig. 2. The schematic illustration of a sensing thin film.

can be expressed as [26]

$\frac{\partial C}{\partial t}=D \frac{\partial^{2} C}{\partial x^{2}}-\frac{\partial N}{\partial t}$

where $C$ is the concentration of gas which is a function of position $(x)$ and time $(t), D$ the diffusivity of gas molecule inside a solid thin film, and $N$ the concentration of the adsorbed species. Under such a condition, the local equilibrium can be assumed to exist between the free and the adsorbed molecules. The scheme of sensing film is illustrated in Fig. 2, in which the length and the thickness of the film are denoted by $l$ and $x_{0}$, respectively. The following two sensing cases are discussed separately:

(i) Linear case (in lower concentration sensing)

In the case of low concentration sensing, the adsorption is proportional to the free concentration, or

$N=k C$

Eq. (1) can be further simplified by substituting Eq. (2) as

$\frac{\partial C}{\partial t}=D_{\mathrm{e}} \frac{\partial^{2} C}{\partial x^{2}}$

where $D_{\mathrm{e}}$ is the effective diffusivity and is defined by $D_{\mathrm{e}}=\frac{D}{1+k}$, the initial and boundary conditions are

$t=0, \quad 0 \leq x \leq x_{0}, \quad C(x, 0)=0$

$t>0, \quad x=x_{0}, \quad C\left(x_{0}, t\right)=C_{0}$

$x=0, \quad \frac{\partial C}{\partial x}=0$

The exact solution to Eqs. (3)-(6) can be found [26] as

$$
\begin{aligned}
C(x, t)= & C_{0}-\frac{4 C_{0}}{\pi} \sum_{n=0}^{\infty} \frac{(-1)^{n}}{(2 n+1)} \\
& \times \exp \left[\frac{-D(2 n+1)^{2} \pi^{2}}{4 x_{0}^{2}} t\right] \cos \left(\frac{(2 n+1) \pi x}{2 x_{0}}\right)
\end{aligned}
$$

(ii) Nonlinear case (in higher concentration sensing) 
When operating in a higher concentration environment, the gas adsorption follows the Freundlich isotherm model

$N=k C^{\gamma}$

where $\gamma$ is a characteristic constant or the so-called power index (sensitivity) of the system, which accounts for the relationship between the adsorption coverage and the gas concentration. For a higher concentration case, the adsorption of gas depends weakly on the gas concentration, thus the $\gamma$ value is usually less than unity. The larger the $\gamma$ is, the stronger the adsorption dependence is on the gas concentration during the adsorption process. For films with a larger $\gamma$ value, films are more sensitive in response to the variation of gas concentration. Substituting Eq. (8) into Eq. (1) and assuming that $\frac{\partial C}{\partial t} \ll \frac{\partial N}{\partial t}$ (i.e., $k \gg 1$ ), Eq. (1) can be rewritten as

$$
\frac{\partial N}{\partial t}=\frac{\partial}{\partial x}\left[\frac{D}{\gamma} k^{-1 / \gamma} N^{(1-\gamma) / \gamma} \frac{\partial N}{\partial x}\right]
$$

or

$\frac{\partial N}{\partial t}=D k^{-1 / \gamma} \frac{\partial^{2} N^{1 / \gamma}}{\partial x^{2}}$

The effective diffusivity $\left(D_{\mathrm{e}}\right)$ can be expressed as

$D_{\mathrm{e}}=\frac{D}{\gamma} k^{-1 / \gamma} N^{(1-\gamma) / \gamma}$

which is a function of $N$ and $\gamma$. If $\gamma<1, D_{\mathrm{e}}$ increases as the adsorbed concentration $N$ increases. When $\gamma>1, D_{\mathrm{e}}$ decreases as the adsorbed concentration $N$ increases. Eq. (10) can be further simplified as

$$
\frac{\partial S}{\partial T}=\frac{\partial^{2} S^{1 / \gamma}}{\partial X^{2}}
$$

The associated initial and boundary conditions are

$T=0, \quad 0 \leq X \leq 1, \quad S(X, 0)=0$

$T>0, \quad X=1, \quad S(1, T)=1$

$X=0, \quad \frac{\partial S}{\partial X}=0$

where the dimensionless groups $S, T$, and $X$ are defined as

$$
\begin{aligned}
S & =\frac{N}{N_{0}} \\
T & =\frac{t}{\tau} \\
X & =\frac{x}{x_{0}}
\end{aligned}
$$

and

$\tau=\frac{k x_{0}^{2} C_{0}^{\gamma-1}}{D}$ or

$\tau=\frac{N_{0}}{C_{0}} \frac{x_{0}^{2}}{D}$

where $N_{0}$ is the maximum adsorbed concentration and is normally called trap concentration. Numerical solution for Eqs. (12)-(15) has been proposed [27] by considering the nonlinear diffusion model.

The nonlinear Freundlich isotherm in Eq. (8) is adapted as a general case for a p-type PbPc thin film which undergoes a rapid adsorption and generates holes that increase the conductivity

$$
\begin{aligned}
\mathrm{NO}+\mathrm{PbPc} & \rightleftharpoons\left[\mathrm{NO}^{-} \mathrm{PbPc}^{+}\right] \\
& \rightleftharpoons\left[\mathrm{NO}^{-} \mathrm{PbPc}\right]+\text { hole }
\end{aligned}
$$

Assuming the conductivity is proportional to the adsorbed amount $(N)$ or the coverage $(\theta)$ as

$\sigma \propto N \propto \theta \propto k C^{\gamma}$

the conductivity $\left(\sigma[=] \Omega^{-1} \mathrm{~cm}^{-1}\right.$ ) of p-type semiconductor is

$\sigma=\mu q n$

where $\mu$ is the hole mobility $\left(\mathrm{cm}^{2} / \mathrm{V} \mathrm{s}\right), q$ the charge of a single hole (C/no.), and $n$ the carrier density (no. $/ \mathrm{cm}^{3}$ ). Therefore, the drift current density

$J=\sigma E=\mu q n E$

The current response is then written by using Eq. (22) and applying the surface integration to the closed electrode surface

$\Delta I=k \mu q \int_{s}[C(x, y, z, t)]^{\gamma} E(x, y, t) \mathrm{d} s$

Eq. (25) can be simplified further by considering a onedimensional diffusion and that $\mathrm{d} s \approx l \mathrm{~d} x$ if the dimension in $y$-direction ( $l$, the length of the film shown in Fig. 2) is kept constant

$\Delta I=9 k \mu q l \int_{0}^{x_{0}}(C(x, t))^{\gamma} E(x) \mathrm{d} x$

where constant 9 in Eq. (26) represents nine crosssectional planes in the design of an interdigitated gold electrode, as shown in Fig. 1. The electric field distribution shown in Eq. (26) is further simplified by Gardner [28]

$E(x, t) \approx E(x)=\frac{V}{\pi}\left[\left(x-x_{0}\right)^{2}+\frac{w^{2}}{4}\right]^{-1 / 2}$

where $V$ is the applied potential and $w$ the distance between two electrodes.

For gas sensing in a higher concentration range, a nonlinear diffusion $(\gamma \neq 1)$ arises. Substituting Eq. (27) into 
Eq. (26) gives

$$
\Delta I=9 k \mu q l \frac{V}{\pi} \int_{0}^{x_{0}}(C(x, t))^{\gamma}\left(\left(x-x_{0}\right)^{2}+\frac{w^{2}}{4}\right)^{-1 / 2} \mathrm{~d} x
$$

or

$$
\Delta I=9 \mu q l \frac{V}{\pi} N_{0} \int_{0}^{x_{0}} S(x, t)\left(\left(x-x_{0}\right)^{2}+\frac{w^{2}}{4}\right)^{-1 / 2} \mathrm{~d} x
$$

where the profile of $S(x, t)$ is numerically obtained by solving Eqs. (12)-(15). Since $x_{0} \ll w$, the maximum current response $\left(\Delta I_{\max }\right)$ can be obtained by letting $t \rightarrow \infty$, or

$$
\Delta I_{\max } \approx 18 k \mu q l \frac{V}{\pi w} C_{0}^{\gamma} x_{0}
$$

As for sensing in a lower concentration range, $\gamma$ reaches unity. By substituting Eq. (7) into Eq. (28), the current response can be simplified as

$$
\begin{aligned}
\Delta I= & 9 k \mu q l \frac{V}{\pi} \int_{0}^{x_{0}}\left\{C_{0}-\frac{4 C_{0}}{\pi} \sum_{n=0}^{\infty} \frac{(-1)^{n}}{(2 n+1)}\right. \\
& \left.\times \exp \left(\frac{-D(2 n+1)^{2} \pi^{2}}{4 x_{0}^{2}} t\right)\right\} \cos \left(\frac{(2 n+1) \pi x}{2 x_{0}}\right) \\
& \times\left(\left(x-x_{0}\right)^{2}+\frac{w^{2}}{4}\right)^{-1 / 2} \mathrm{~d} x
\end{aligned}
$$

According to the result derived in Eq. (30), the characteristic power index $(\gamma)$ for the NO-PbPc system can be obtained by plotting $\log \left(\Delta I_{\max }\right)$ against $\log \left(C_{0}\right)$.

\section{Results and discussions}

\subsection{Operating temperature}

It is well known that the conductivity of a semiconductor is affected by the surrounding temperature. Generally, the increase in temperature leads to a higher conductivity, because the increase in thermal motion enhances the movement of the carriers within the semiconductor. On the other hand, the increase in temperature retards the gas adsorption, which brings more holes when adsorption occurs. Because of the two competing factors, an optimum temperature will be reached. The result for a 100-nm PbPc thin film exposed to 100-ppm NO for $25 \mathrm{~min}$ at different temperatures is shown in Fig. 3. The optimum temperature is $170^{\circ} \mathrm{C}$, at which the maximum current response is obtained. For the rest of the experiments, the temperature is thus fixed at $170^{\circ} \mathrm{C}$.

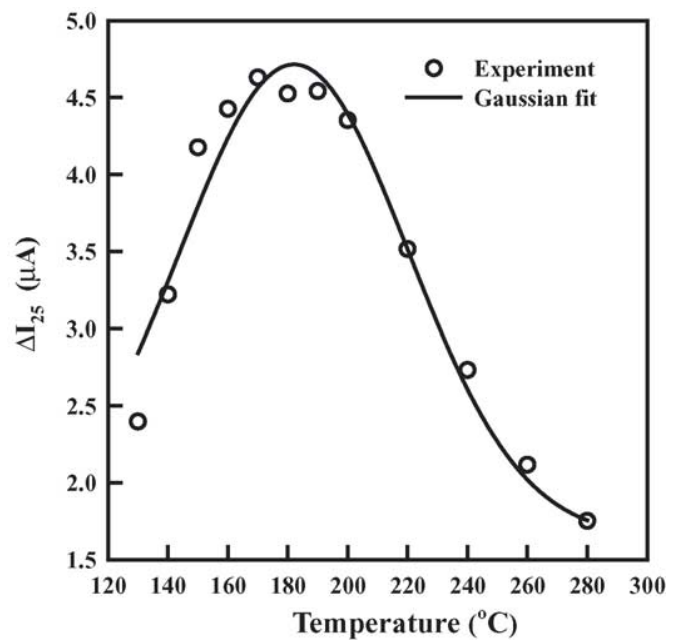

Fig. 3. The maximum current response of a PbPc film exposed to $100 \mathrm{ppm}$ $\mathrm{NO}$ at different temperatures for $25 \mathrm{~min}$. Film thickness: $100 \mathrm{~nm}$, evaporation rate: $0.5 \mathrm{~nm} / \mathrm{s}$, substrate temperature: $25^{\circ} \mathrm{C}$, flow rate: $200 \mathrm{ml} / \mathrm{min}$.

\subsection{Calibration curve for an untreated film}

Typical sensing curve for a 300-nm untreated, freshly made $\mathrm{PbPc}$ thin film exposed to various $\mathrm{NO}$ concentrations is shown in Fig. 4. The response time for the adsorption is faster than that of the desorption process. The steady-state current values for a 300-nm PbPc thin film together with those of 200 and $100 \mathrm{~nm} \mathrm{PbPc}$ thin films obtained under various NO exposures are replotted against the bulk NO concentration $\left(C_{0}\right)$ in Fig. 5. The slope in the full log plot is the value of $\gamma$ mentioned in Eq. (30). It can be found that the slopes for all three film thicknesses $(100,200$, and $300 \mathrm{~nm})$, as summarized in Table 1, are roughly the same. It is noted that as the film becomes thicker, the response current is larger as well. This is because a thicker film possesses larger cross-sectional area which is perpendicular to the direction of the electric current.

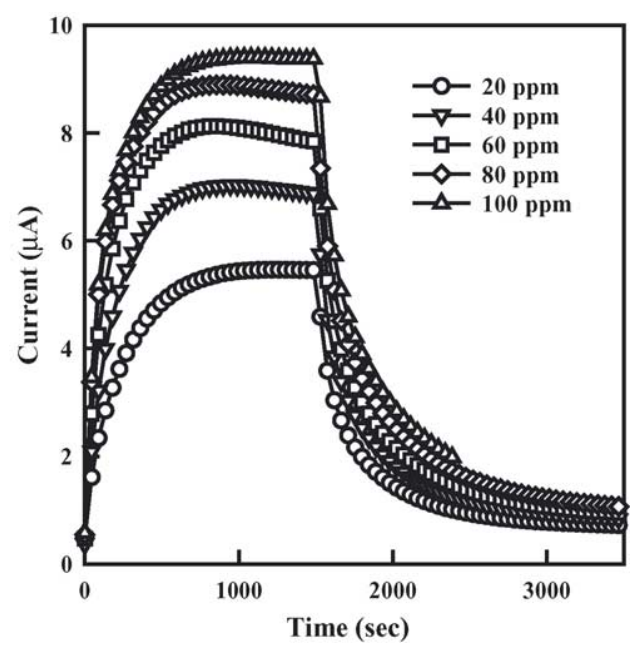

Fig. 4. The current responses of a PbPc film exposed to different NO concentrations. Film thickness: $300 \mathrm{~nm}$, evaporation rate: $0.5 \mathrm{~nm} / \mathrm{s}$, substrate temperature: $25^{\circ} \mathrm{C}$, operating temperature: $170^{\circ} \mathrm{C}$, flow rate: $200 \mathrm{ml} / \mathrm{min}$. 


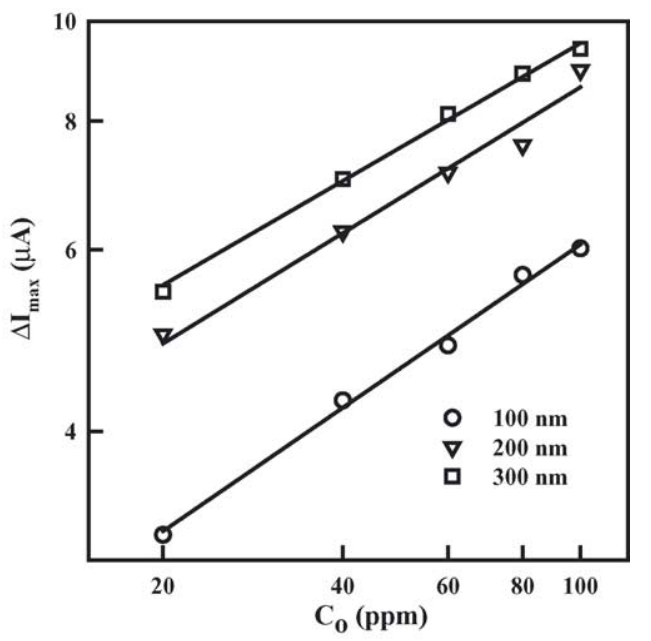

Fig. 5. The full log plot of the maximum current change vs. inlet concentrations for different thicknesses of PbPc films. Evaporation rate: $0.5 \mathrm{~nm} / \mathrm{s}$, substrate temperature: $25^{\circ} \mathrm{C}$, operating temperature: $170^{\circ} \mathrm{C}$, flow rate: $200 \mathrm{ml} / \mathrm{min}$

Thus, a larger current is expected according to the following equation when films are applied at the same potential $(10 \mathrm{~V}$ DC):

$\frac{I}{V}=G=\sigma \frac{A}{l}$

where $G$ is the conductance, $\sigma$ the conductivity, $A$ the crosssectional area perpendicular to the electric current, and $l$ the length along the direction of the electric current.

\subsection{Choice of solvent}

Various solvents are chosen in the post-treatment for a 100-nm PbPc thin film, as shown in Fig. 6. Solvents such as ethanol (EtOH), water, acetonitrile, acetone, isopropanol, and $n$-hexane are used. It is noticed that all post-treated films possess lower currents as compared to that of the untreated one, which results from the morphological reformation from an amorphous to a more crystal structure that will be discussed in a later section. The other reason for lower response current is presumably due to the strong adsorption between $\mathrm{PbPc}$ and solvent molecules rather than NO molecules, even when operated at an elevated temperature of $170^{\circ} \mathrm{C}$. Another possible reason may be due

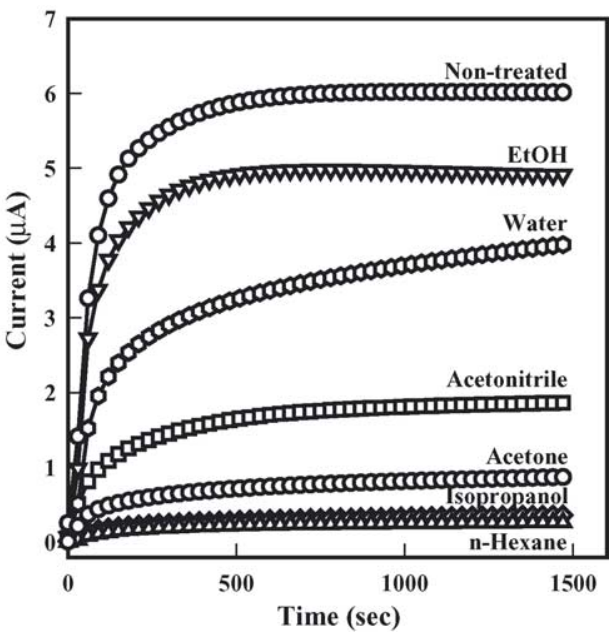

Fig. 6. The current responses of untreated and solvent-treated PbPc films exposed to $100 \mathrm{ppm}$ NO. Film thickness: $100 \mathrm{~nm}$, evaporation rate: $0.5 \mathrm{~nm} / \mathrm{s}$, substrate temperature: $25^{\circ} \mathrm{C}$, operating temperature: $170^{\circ} \mathrm{C}$, flow rate: $200 \mathrm{ml} / \mathrm{min}$. Except for the non-treated film, all evaporated films have been treated in $\mathrm{EtOH}$, water, acetonitrile, acetone, isopropanol, and $n$-hexane vapors for $24 \mathrm{~h}$.

to the dissolution of PbPc films in organic vapor environments.

Among all solvent-treated films, the one with ethanoltreated possesses the highest current response. Moreover, a faster response time is also achieved as compared to the untreated one. This brings a promising possibility for sensing applications.

\subsection{Sensing performance with ethanol-treated films}

From previous discussion, our attention is focused on analyzing the performance of ethanol-treated $\mathrm{PbPc}$ thin films. A typical sensing curve for a 300-nm film is shown in Fig. 7. The corresponding full log plot of the maximum current change versus inlet NO concentration for the ethanol-treated films is shown in Fig. 8. The fitted $\gamma$ values for 100, 200, and $300 \mathrm{~nm}$ PbPc films are 0.65, 0.39, and 0.66 (also summarized in Table 1), respectively. Except for film thickness of $200 \mathrm{~nm}, \gamma$ value increases almost twice as compared to those of untreated films. This means that the Freundlich adsorption isotherm model is altered after ethanol-treatment. The increase in $\gamma$ value enhances the adsorption process (as Eq.

Table 1

A comparison of maximum current and $\gamma$ values between $\mathrm{PbPc}$ films without treatment and with EtOH treatment

\begin{tabular}{|c|c|c|c|c|c|c|c|}
\hline & $\mathrm{PbPc}$ films & Maximu & $t(\mu \mathrm{A})$ & & & & $\gamma$ \\
\hline & & $20 \mathrm{ppm}$ & $40 \mathrm{ppm}$ & $60 \mathrm{ppm}$ & $80 \mathrm{ppm}$ & $100 \mathrm{ppm}$ & \\
\hline $100 \mathrm{~nm}$ & Untreated (amorphous) & 3.17 & 4.28 & 4.85 & 5.67 & 6.02 & 0.38 \\
\hline & Treated with EtOH ( $\alpha$ phase) & 0.09 & 0.13 & 0.18 & 0.21 & 0.25 & 0.65 \\
\hline $200 \mathrm{~nm}$ & Untreated (amorphous) & 4.97 & 6.25 & 7.13 & 7.58 & 8.97 & 0.33 \\
\hline & Treated with EtOH ( $\alpha$ phase) & 0.53 & 0.67 & 0.79 & 0.90 & 0.99 & 0.39 \\
\hline $300 \mathrm{~nm}$ & Untreated (amorphous) & 5.46 & 7.03 & 8.13 & 8.89 & 9.40 & 0.34 \\
\hline & Treated with EtOH ( $\alpha$ phase) & 1.40 & 2.11 & 2.53 & 3.25 & 3.87 & 0.66 \\
\hline
\end{tabular}




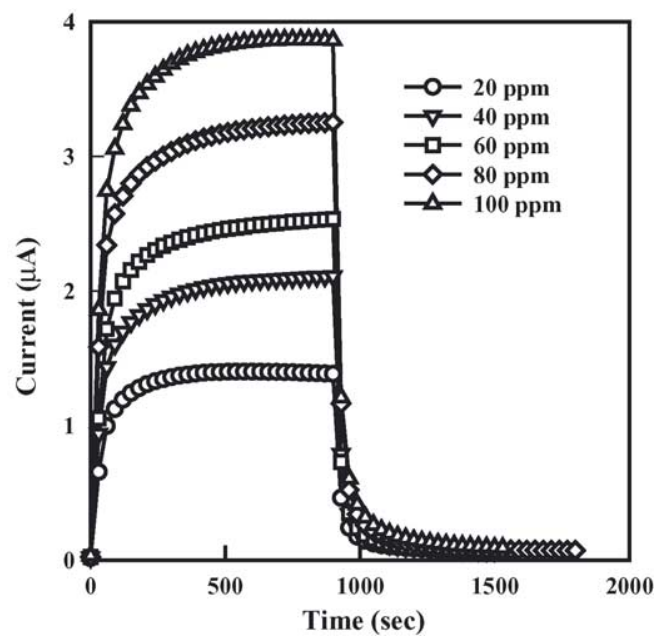

Fig. 7. The current responses of an EtOH-vapor-treated $\mathrm{PbPc}$ film exposed to different NO concentrations. Film thickness: $300 \mathrm{~nm}$, evaporation rate: $0.5 \mathrm{~nm} / \mathrm{s}$, substrate temperature: $25^{\circ} \mathrm{C}$, operating temperature: $170^{\circ} \mathrm{C}$, flow rate: $200 \mathrm{ml} / \mathrm{min}$.

(8) depicts), which results in a faster response time during the sensing process.

\subsection{Morphology}

Fig. 9 is the XRD analyses for a 300-nm PbPc thin film before and after ethanol treatment. The increase in the XRD intensity represents the transformation from amorphous to $\alpha$ phase crystal structure $\left(2 \theta=12.5-12.7^{\circ}\right)$. The SEM images, as shown in Fig. 10, also support the crystalline formation after $24-\mathrm{h}$ post-treatment. The increase grain size after posttreatment could cause decrease in the adsorptive surface area which offers binding sites for NO adsorption. That is one of the reasons that the current responses are always lower for solvent-treated films as compared to those of untreated films in Fig. 6.

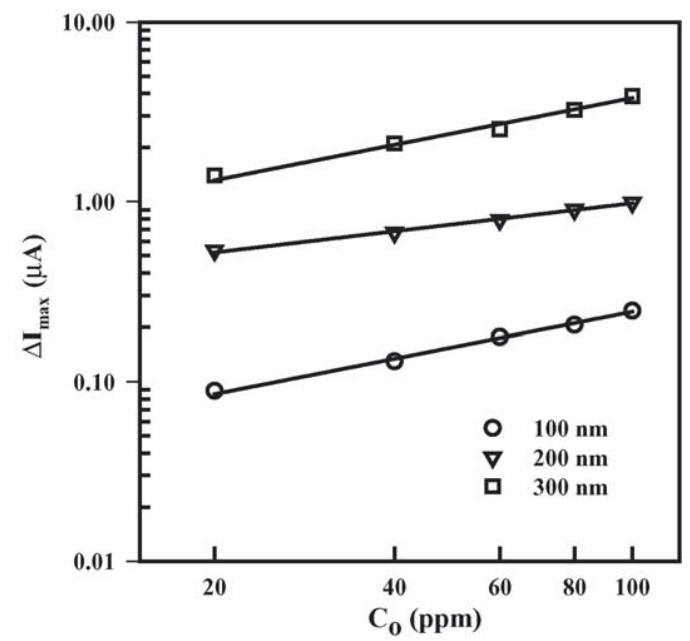

Fig. 8. The full log plot of the maximum current change vs. inlet concentrations of EtOH-vapor-treated PbPc films. Evaporation rate: $0.5 \mathrm{~nm} / \mathrm{s}$, substrate temperature: $25^{\circ} \mathrm{C}$, operating temperature: $170^{\circ} \mathrm{C}$, flow rate: $200 \mathrm{ml} / \mathrm{min}$.

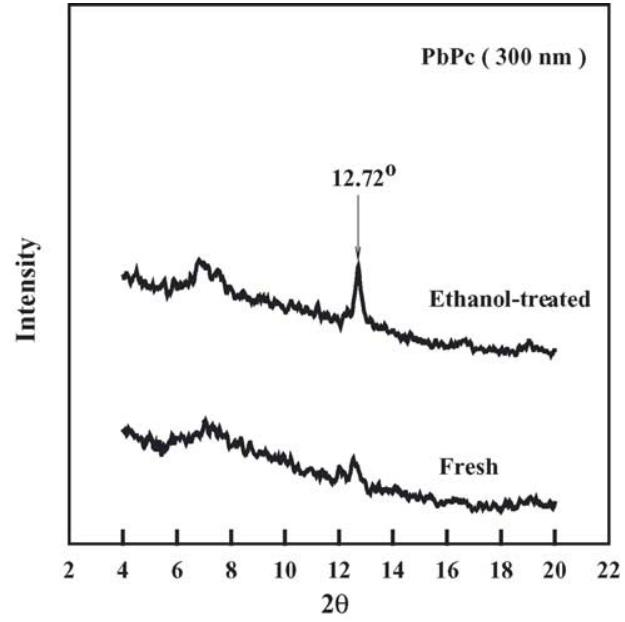

Fig. 9. XRD patterns for $\mathrm{PbPc}$ films deposited on $\mathrm{Al}_{2} \mathrm{O}_{3}$ substrate with a film thickness of $300 \mathrm{~nm}$ before and after ethanol-treatment for $24 \mathrm{~h}$

\subsection{Data fitting}

Based on Eqs. (12) and (29), an attempt has been made to fit the sensing data by a numerical means. Several parameters are required for the numerical procedure, such as $\gamma$ (obtained from Figs. 5 and 8 and summarized in Table 1), $\mu$
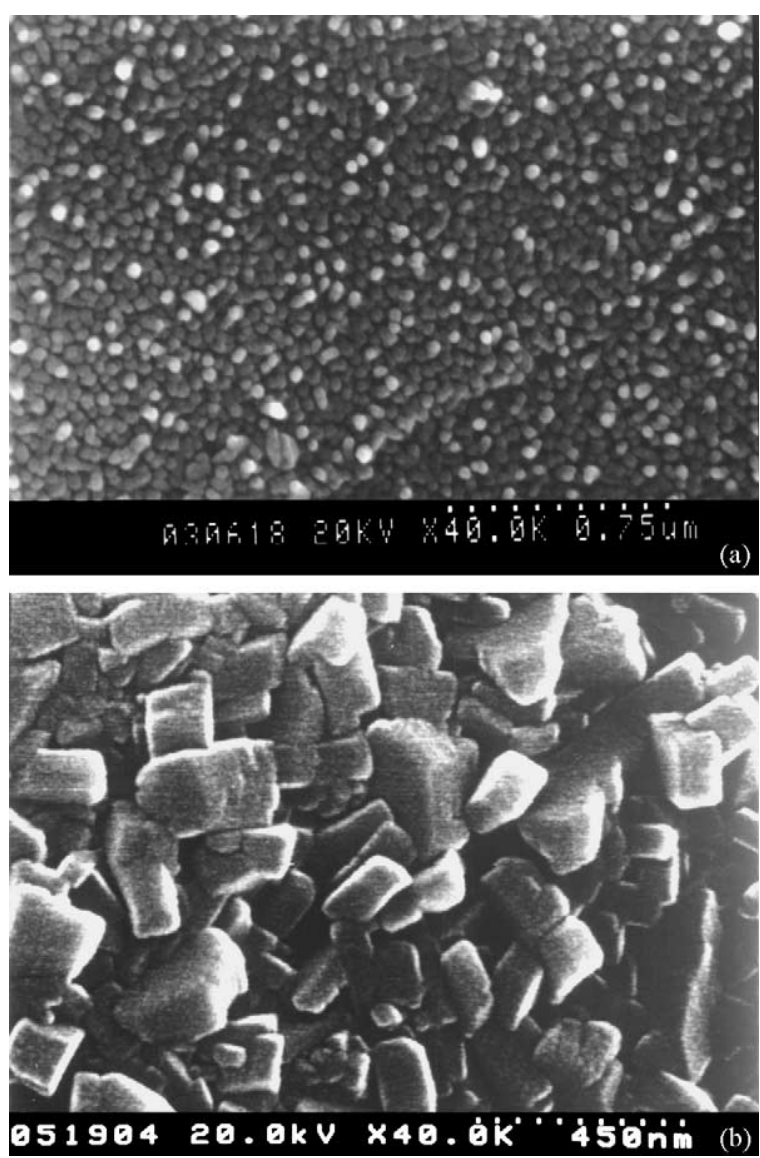

Fig. 10. SEM micrographs of a 300-nm PbPc film (a) before and (b) after ethanol-treatment for $24 \mathrm{~h}$. 


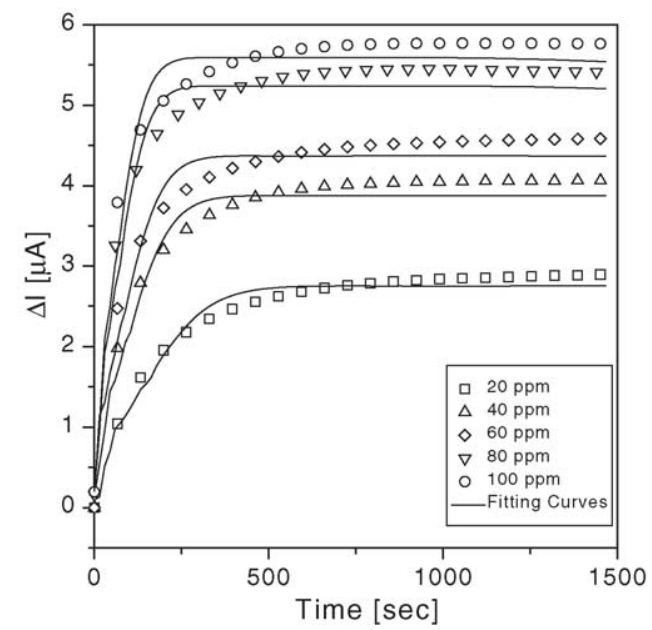

Fig. 11. A comparison of measured currents (empty shape) and the fitting curves (continuous curve) for a 100-nm untreated PbPc thin film exposed to different concentrations of $\mathrm{NO}$ gas.

$\left(=6.0 \times 10^{-10} \mathrm{~m}^{2} / \mathrm{V} \mathrm{s}[29]\right), l(=7 \mathrm{~mm}), q\left(=1.6 \times 10^{-19} \mathrm{C}\right), V$ $(=10 \mathrm{~V})$. The maximum adsorbed concentration or trap concentration, $N_{0}$, can be obtained from Eq. (8) when concentration is $C_{0}$. The fitting results, both for untreated and treated films, are shown in Figs. 11 and 12, respectively. The calculated parameters both for untreated and treated films are summarized in Tables 2 and 3, respectively.

According to the fitting data, it can be found that the value of $N_{0}$ (the trap concentration) for an ethanol-treated film is smaller than that of the untreated one. This is consistent with the morphological evidence, because the surface area available for adsorption after ethanol-treatment is smaller due to grain growth and so does the trap concentration. Since our analyses are based on Eq. (1), which assumes a faster adsorption process, the time needed to reach steady state is purely

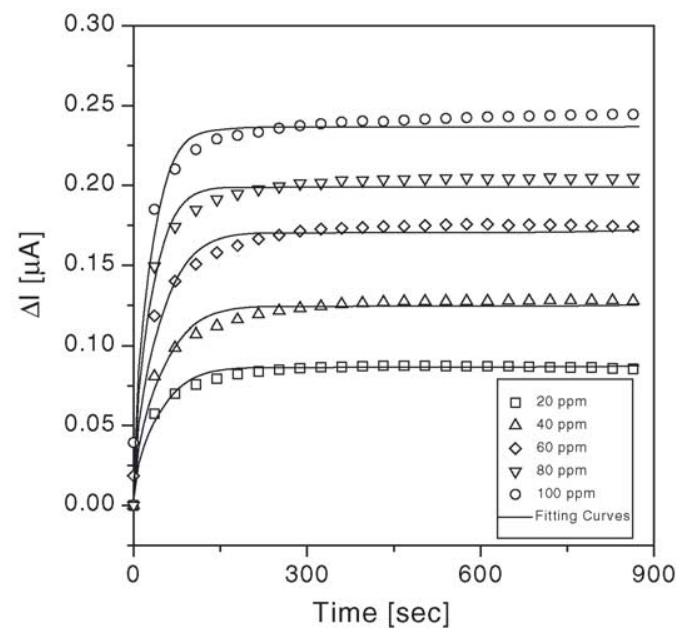

Fig. 12. A comparison of measured currents (empty shape) and the fitting curves (continuous curve) for a 100-nm ethanol-vapor-treated PbPc thin film exposed to different concentrations of NO gas.

due to the diffusion inside a PbPc thin film. Thus, a response time, $\tau_{80}$, which is defined as the time needed to attain $80 \%$ of its steady-state value, can be determined from the fitting results in Figs. 11 and 12. The diffusion depth at $\tau_{80}$ is denoted as $\delta_{80}$, which can be obtained through Eq. (20). Similarly, an averaged diffusion depth can also be evaluated by $\sqrt{D_{\mathrm{e}} \tau_{80}}$, whose values are generally on the same order of magnitude as that of $\delta_{80}$. The diffusion depths are different when comparing the film treated with ethanol and that without treatment. The treatment does result in the morphology change. As a matter of fact, the calculated diffusion depth $\left(\delta_{80}\right)$ is on the same order of magnitude as the averaged diffusion depth $\left(\sqrt{D_{\mathrm{e}} \tau_{80}}\right)$, which suggests that the ethanol treatment process may not be able to alter the diffusion mechanism, but does improve the adsorption kinetics (larger $\gamma$ value).

Table 2

The fitting values of $k, D, N_{0}, D_{\mathrm{e}}, \tau_{80}, \delta_{80}$, and $\sqrt{D_{\mathrm{e}} \tau_{80}}$ for a fresh PbPc thin film

\begin{tabular}{|c|c|c|c|c|c|c|c|}
\hline$C_{0}(\mathrm{ppm})$ & $k\left(\left(\mathrm{~m}^{-3}\right)^{0.62}\right)$ & $D\left(\mathrm{~m}^{2} / \mathrm{s}\right)$ & $N_{0}\left(\mathrm{~m}^{-3}\right)$ & $D_{\mathrm{e}}\left(\mathrm{m}^{2} / \mathrm{s}\right)$ & $\tau_{80}(\mathrm{~s})$ & $\delta_{80}(\mathrm{~m})$ & $\sqrt{D_{\mathrm{e}} \tau_{80}}(\mathrm{~m})$ \\
\hline 20 & $1.92 \times 10^{18}$ & $5.18 \times 10^{-12}$ & $1.42 \times 10^{26}$ & $0.49 \times 10^{-16}$ & 318 & $7.74 \times 10^{-8}$ & $12.48 \times 10^{-8}$ \\
\hline 40 & $2.08 \times 10^{18}$ & $5.37 \times 10^{-12}$ & $2.01 \times 10^{26}$ & $0.73 \times 10^{-16}$ & 219 & $7.76 \times 10^{-8}$ & $12.60 \times 10^{-8}$ \\
\hline 60 & $2.01 \times 10^{18}$ & $4.59 \times 10^{-12}$ & $2.27 \times 10^{26}$ & $0.83 \times 10^{-16}$ & 201 & $7.93 \times 10^{-8}$ & $12.89 \times 10^{-8}$ \\
\hline 80 & $2.16 \times 10^{18}$ & $5.26 \times 10^{-12}$ & $2.72 \times 10^{26}$ & $1.05 \times 10^{-16}$ & 156 & $7.88 \times 10^{-8}$ & $12.80 \times 10^{-8}$ \\
\hline 100 & $2.12 \times 10^{18}$ & $4.77 \times 10^{-12}$ & $2.90 \times 10^{26}$ & $1.11 \times 10^{-16}$ & 141 & $7.73 \times 10^{-8}$ & $12.51 \times 10^{-8}$ \\
\hline Average & $2.06 \times 10^{18}$ & $5.03 \times 10^{-12}$ & & & & $7.81 \times 10^{-8}$ & $12.66 \times 10^{-8}$ \\
\hline
\end{tabular}

Film thickness $=100 \mathrm{~nm}$, film type $=$ amorphous, $\gamma=0.38$.

Table 3

The fitting values of $k, D, N_{0}, D_{\mathrm{e}}, \tau_{80}, \delta_{80}$, and $\sqrt{D_{\mathrm{e}} \tau_{80}}$ for an EtOH-vapor-treated PbPc thin film

\begin{tabular}{lllllrr}
\hline$C_{0}(\mathrm{ppm})$ & $k\left(\left(\mathrm{~m}^{-3}\right)^{0.35}\right)$ & $D\left(\mathrm{~m}^{2} / \mathrm{s}\right)$ & $N_{0}\left(\mathrm{~m}^{-3}\right)$ & $D_{\mathrm{e}}\left(\mathrm{m}^{2} / \mathrm{s}\right)$ & \multicolumn{1}{c}{$\tau_{80}(\mathrm{~s})$} & $\delta_{80}(\mathrm{~m})$ \\
\hline 20 & $1.54 \times 10^{11}$ & $5.95 \times 10^{-13}$ & $4.49 \times 10^{24}$ & $1.06 \times 10^{-16}$ & 90 & $7.84 \times 10^{-8}$ \\
40 & $1.42 \times 10^{11}$ & $3.99 \times 10^{-13}$ & $6.45 \times 10^{24}$ & $0.98 \times 10^{-16}$ & 102 & $8.06 \times 10^{-8}$ \\
60 & $1.49 \times 10^{11}$ & $3.88 \times 10^{-13}$ & $8.84 \times 10^{24}$ & $1.04 \times 10^{-16}$ & 96 & $8.08 \times 10^{-8}$ \\
80 & $1.44 \times 10^{11}$ & $4.75 \times 10^{-13}$ & $1.03 \times 10^{25}$ & $1.46 \times 10^{-16}$ & 71 & $9.00 \times 10^{-8}$ \\
100 & $1.48 \times 10^{11}$ & $4.67 \times 10^{-13}$ & $1.23 \times 10^{25}$ & $1.51 \times 10^{-16}$ & 67 & $8.10 \times 10^{-8}$ \\
Average & $1.47 \times 10^{11}$ & $4.65 \times 10^{-8}$ & & & $10.18 \times 10^{-8}$ \\
\hline
\end{tabular}

Film thickness $=100 \mathrm{~nm}$, film type $=\alpha$ phase, $\gamma=0.65$. 
All the fitting values shown in Tables 2 and 3 are parts of Eqs. (1) and (8), which involve a diffusion condition coupled with a nonlinear adsorption. Therefore these values can be divided into two categories. One is for the adsorption kinetics, such as the proportional constant for adsorption $(k)$ and the adsorption dependency $(\gamma)$. The other is for the diffusion process, including diffusivity ( $D$ and $D_{\mathrm{e}}$ ), diffusion depth ( $\delta_{80}$ and $\sqrt{D_{\mathrm{e}} \tau_{80}}$ ), and diffusion time $\left(\tau_{80}\right)$. Each value is essential to evaluate a sensing system involving both diffusion and adsorption.

\section{Conclusions}

In this work, nitric oxide (NO) sensing using $\mathrm{PbPc}$ thin films is studied. An optimized temperature of $170^{\circ} \mathrm{C}$ was selected at which the highest current response was attained. Ethanol vapor was found to be the most promising solvent for post-treating PbPc thin films. It was found that the response current after ethanol treatment was lower than that of the untreated one, because of surface area reduction caused by $\alpha$-phase crystal structure formation. Based on a nonlinear diffusion/adsorption theory, the slope of the calibration curve in a full $\log$ plot of $\Delta I_{\max }$ versus $C_{0}$ gives the value of $\gamma$, which is a characteristic constant in characterizing film's properties. The $\gamma$ value for ethanol-treated film is roughly double when compared to that of the untreated one. This indicates an improved adsorption property (even though the surface area is fewer on the treated film). A higher trap concentration $\left(N_{0}\right)$ is obtained, which corresponds to a higher surface area for the untreated film. The calculated diffusion depth, $\delta_{80}$, is close to the averaged diffusion depth $\sqrt{D_{\mathrm{e}} \tau_{80}}$, which suggests that the diffusion process does determine the sensing process. This supports the faster adsorption process assumption made at the beginning of the theoretical section.

\section{Acknowledgements}

This work was sponsored by the National Research Council of the Republic of China under grant numbers NSC 922214-E-002-037 and NSC 93-ET-7-002-005-ET.

\section{References}

[1] P.R. Somani, S. Radhakrishnan, Electrochromic materials and devices: present and future, Mater. Chem. Phys. 77 (2002) 117-133.

[2] C.-L. Lin, C.-C. Lee, K.-C. Ho, Spectroelectrochemical studies of manganese phthalocyanine thin films for applications in electrochromic devices, J. Electroanal. Chem. 524-525 (2002) 81-89.

[3] W. Hu, M. Matsumura, Structure and thickness dependence of $\mathrm{p}-\mathrm{n}$ heterojunction solar cells based on copper phthalocyanine and perylene pigments, J. Phys. D: Appl. Phys. 37 (2004) 1434-1438.

[4] A.W.J. Cranny, J.K. Atkinson, A comparison of thick- and thin-film gas-sensitive organic semiconductor compounds, Sens. Actuators B: Chem. 4 (1991) 169-174.
[5] C. Hamann, A. Mrwa, M. Muller, Lead phthalocyanine thin films for $\mathrm{NO}_{2}$ Sensors, Sens. Actuators B: Chem. 4 (1991) 73-78.

[6] A. Belghachi, R.A. Collins, The effects of humidity on phthalocyanine $\mathrm{NO}_{2}$ and $\mathrm{NH}_{3}$ sensors, J. Phys. D: Appl. Phys. 23 (1990) 223-227.

[7] R.C. Weaver, J.D. Wright, Interaction of electron-accepting vapours with phthalocyanine and related compounds: a novel calorimetric study, Sens. Actuators B: Chem. 4 (1991) 505-510.

[8] J.W. Gardner, M.Z. Iskandarani, Effect of electrode geometry on gas sensitivity of lead phthalocyanine thin films, Sens. Actuators B: Chem. 9 (1992) 133-142.

[9] D. Campbell, R.A. Collins, The effect of surface topography on the sensitivity of lead phthalocyanine thin films to nitrogen dioxide, Phys. Stat. Sol. A 152 (1995) 431-442.

[10] Y. Sadaoka, Y. Sakai, T.A. Jones, W. Göpel, Effects of oxidizing gases on semiconductivity and thermal stability of phthalocyanine thin films, J. Mater. Sci. 25 (1990) 3024-3028.

[11] C. Hamann, A. Mrwa, M. Müller, Lead phthalocyanine thin films for $\mathrm{NO}_{2}$ sensors, Sens. Actuators B: Chem. 4 (1991) 73-78.

[12] A. Heilmann, V. Lantto, M. Müller, C. Hamann, $\mathrm{NO}_{2}$ monitoring as an air pollutant using lead phthalocyanine thin film sensors, Sens. Actuators B: Chem. 7 (1992) 522-525.

[13] M. Rapp, D. Bunz, I. Kabbe, M. Von Schickfus, S. Hunklinger, A new high-frequency high-sensitivity SAW device for $\mathrm{NO}_{2}$ gas detection in the sub-ppm range, Sens. Actuators B: Chem. 4 (1991) 103-108.

[14] A. Wilson, J.D. Wright, A.V. Chadwick, A microprocessor-controlled nitrogen dioxide sensing system, Sens. Actuators B: Chem. 4 (1991) 499-504.

[15] J.E. Darby, R.J. Mcilroy, G. Revell, S.C. Thorpe, A. Wilson, Effect of electron-acceptor gases on lead oxide and lead phthalocyanine thin films: a comparative study, Sens. Actuators B: Chem. 3 (1991) $157-160$.

[16] A.K. Abass, A. Krier, R.A. Collins, The influence of chlorine on the electrical properties of lead phthalocyanine thin films gas sensors, J. Phys. D: Appl. Phys. 26 (1993) 1120-1124.

[17] R.A. Collins, A.K. Abass, A. Krier, The infrared response of lead phthalocyanine thin films to chlorine and iodine doping, Thin Solid Films 239 (1994) 268-271.

[18] J.P. Lukaszewicz, $\mathrm{LaF}_{3}$-based oxygen sensor using $\mathrm{Pb}$ phthalocyanine electrode for quick response at room temperature, Sens. Actuators B: Chem. 9 (1992) 55-58.

[19] S. Kanefusa, M. Nitta, The detection of $\mathrm{H}_{2}$ gas by metal phthalocyanine-based gas sensors, Sens. Actuators B: Chem. 9 (1992) 85-90.

[20] C.J. Liu, J.J. Shih, Y.H. Ju, Surface morphology and gas-sensing characteristics of nickel phthalocyanine thin films, Sens. Actuators B: Chem. 99 (2004) 344-349.

[21] C. Park, D.H. Yun, S.-T. Kim, Y.W. Park, Enhancement of the $\mathrm{NO}_{2}$ sensing capability of copper phthalocyanine by measuring the relative resistance change, Sens. Actuators B: Chem. 30 (1996) 23-27.

[22] M. Passard, C. Maleysson, A. Pauly, S. Dogo, J.-P. Germain, J.-P. Blanc, Gas sensitivity of phthalocyanine thin films, Sens. Actuators B: Chem. 18-19 (1994) 489-492.

[23] M.S. Nieuwenhuizen, A.J. Nederlof, A silicon-based SAW chemical sensor for $\mathrm{NO}_{2}$ by applying a silicon nitride passivation layer, Sens. Actuators B: Chem. 9 (1992) 171-176.

[24] K.-C. Ho, Y.-H. Tsou, Chemiresistor-type NO gas sensor based on nickel phthalocyanine thin films, Sens. Actuators B: Chem. 77 (2001) 253-259.

[25] K.-C. Ho, J.-Y. Liao, $\mathrm{NO}_{2}$ gas sensing based on vacuum-deposited TTF-TCNQ thin films, Sens. Actuators B: Chem. 93 (2003) 370-378.

[26] J. Crank, The Mathematics of Diffusion, 2nd ed., Oxford University Press, Oxford, 1975, pp. 326-329.

[27] J.W. Gardner, A nonlinear diffusion-reaction model of electrical conduction in semiconductor gas sensors, Sens. Actuators B: Chem. 1 (1990) 166-170. 
[28] J.W. Gardner, A diffusion-reaction model of electrical conduction in tin oxide gas sensors, Semicond. Sci. Technol. 4 (1989) 345-350.

[29] A. Ahmad, R.A. Collins, The effect of oxygen on the electrical characteristics of triclinic lead phthalocyanine, Thin Solid Films 217 (1992) 75-82.

\section{Biographies}

Kuo-Chuan Ho received BS and MS degrees in chemical engineering from National Cheng Kung University, Tainan, Taiwan, in 1978 and 1980, respectively. In 1986, he received the $\mathrm{PhD}$ degree in chemical engineering at the University of Rochester. The same year he joined PPG Industries, Inc., first as a Senior Research Engineer and then, from 1990 until 1993, as a Research Project Engineer. He has worked on the electrochemical properties of various electrode materials, with emphasis on improving the performances of sensor devices. Following a 6-year industrial career at PPG Industries, Inc., he joined his alma mater at National Cheng Kung University in 1993 as an Associate Professor in the Chemical Engineering Department. In 1994, he moved to the Department of
Chemical Engineering at National Taiwan University. Currently, he is a Professor jointly appointed by the Department of Chemical Engineering and Institute of Polymer Science and Engineering at National Taiwan University.

Chun-Ming Chen received his BS degree in chemical engineering from National Taiwan Institute of Technology (currently, renamed as National Taiwan University of Science and Technology), Taipei, Taiwan, in 1996. He received MS degree in chemical engineering from National Taiwan University in 1998. Currently, he is a Technology Development Engineer at SST Taiwan Ltd., Hsinchu, Taiwan. He is working on new flash memory development and manufacturing. His research interest mainly is in sensor and high functionality flash memory applications.

Jung-Yu Liao received his BS degree in chemical engineering from National Taiwan University, Taipei, Taiwan, in 1999. He received $\mathrm{PhD}$ degree in chemical engineering from National Taiwan University in 2004. Currently, he is in the army to fulfill the compulsory military service. His research interest mainly surrounds applications of chemically modified electrodes on sensing and electro-optical devices. 